University of Nebraska - Lincoln

DigitalCommons@University of Nebraska - Lincoln

Faculty Publications, Department of Physics and Astronomy

Research Papers in Physics and Astronomy

2003

\title{
Light Storage with Light of Arbitrary Polarization
}

\author{
Hong Gao \\ University of Nebraska - Lincoln \\ Mark Rosenberry \\ University of Nebraska-Lincoln, mrosenberry@siena.edu \\ Herman Batelaan \\ University of Nebraska - Lincoln, hbatelaan@unl.edu
}

Follow this and additional works at: https://digitalcommons.unl.edu/physicsfacpub

Part of the Physics Commons

Gao, Hong; Rosenberry, Mark; and Batelaan, Herman, "Light Storage with Light of Arbitrary Polarization" (2003). Faculty Publications, Department of Physics and Astronomy. 120.

https://digitalcommons.unl.edu/physicsfacpub/120

This Article is brought to you for free and open access by the Research Papers in Physics and Astronomy at DigitalCommons@University of Nebraska - Lincoln. It has been accepted for inclusion in Faculty Publications, Department of Physics and Astronomy by an authorized administrator of DigitalCommons@University of Nebraska Lincoln. 


\title{
Light storage with light of arbitrary polarization
}

\author{
Hong Gao, Mark Rosenberry, and Herman Batelaan \\ Department of Physics and Astronomy, University of Nebraska, Lincoln, 116 Brace Lab, Lincoln, Nebraska 68588-0111
}

(Received 20 December 2002; published 27 May 2003)

\begin{abstract}
We have demonstrated the phase coherence of stored light in $\mathrm{Rb}$ vapor with a completely optical technique. Combining this technique with polarization measurements provides strong evidence that arbitrary polarizations can be stored. The fidelity obtained exceeds $95 \%$ for all polarizations. We view the capability to store polarizations as a first step towards building a quantum memory in such a system.
\end{abstract}

DOI: 10.1103/PhysRevA.67.053807

PACS number(s): 42.50.Gy, 03.67.-a

Storage and recovery of light in an atomic ensemble is an exciting topic in the field of quantum information because photons are excellent long-distance carriers of information, and quantum gates can be realized with atoms [1]. Recently, mapping a polarization state of light onto an atomic spin state via electromagnetically induced transparency (EIT) [2] has been proposed [3-6] and demonstrated [7,8]. Transferring the polarization state of light to another laser (multiplexing) has also been shown [9]. A photon polarization state is a physical realization of a quantum bit (qubit). The general description of a qubit, $\alpha|1\rangle+\beta|0\rangle$, can be mapped onto the Bloch sphere, which for light covers all possible completely polarized states.

In this paper, we address the question of whether or not all polarization states can be stored in an atomic vapor. Light storage experiments $[7,8]$ have focused on atomic $\Lambda$ systems, where $\sigma^{+}$polarized light couples one ground state to the excited state, while $\sigma^{-}$polarized light couples another ground state to the same excited state [Fig. 1(a)]. Slowing of light with linearly polarized light has been studied [10]. In effect, a polarization state corresponding to one point on the Bloch sphere has been stored. Theory predicts [3-5] that other polarizations could be stored using a $\Lambda$ system coupled with orthogonally polarized strong and weak fields.

We demonstrate storage of different polarization states in an atomic $\mathrm{Rb}$ vapor. We first verify experimentally that the phase shift between the control and signal lasers is not affected by storage, which demonstrates optically the coherence of the light storage technique. This is an alternative to the demonstration of coherence control through the use of an external magnetic field [11]. Our optical technique appears to be applicable to the storage of light in an arbitrary medium, such as optically dense crystals [12]. We then demonstrate storage of several elliptical polarizations, beginning with the special case of linearly polarized light. With the reasonable assumptions that our experiment is both cylindrically symmetric and invariant under a parity operation, our measurements can be extended over the entire Bloch sphere, providing strong evidence that all polarization states can be stored.

Our experimental setup is a modified version of that described in Ref. [7]. An extended cavity diode laser with several tens of kiloHertz bandwidth is used as the light source. An acoustic-optical modulator and iris allow for rapid switching of this source. The linearly polarized laser light can be slightly rotated by a fast Pockel's cell to create a weak pulse, which is the signal field $\left(I_{s}, y\right.$ direction). The polar- ization of the weak pulse is perpendicular to that of the remaining light, which serves as the control field $\left(I_{c}, x\right.$ direction). The key modification to our experiment is the addition of a Mach-Zender interferometer. Two polarizing beam splitters (PBS) are used to separate the control and signal beams, pass them along different paths, and recombine them. A piezoelectric transducer (PZT) is connected to the reflector in the signal path giving a phase shift between the two paths [Fig. 1(b)].

Our $\mathrm{Rb}$ cell is $4 \mathrm{~cm}$ long, $2 \mathrm{~cm}$ in diameter, and contains about 5 torr of Helium buffer gas. A solenoid is placed around the $\mathrm{Rb}$ cell to precisely control the static magnetic field along the propagation direction of the laser beam. The solenoid is enclosed within double layered magnetic shielding to ensure long lifetimes of the atomic Zeeman coherence. A small field may be applied by the solenoid to compensate for the residual Earth magnetic field to within $2 \mathrm{mG}$ (the resolution of our probe). This limits the magnetic-field phase shift to $0.03 \mathrm{rad}$. In order to obtain greater atomic density, an Aerobiax heater cable is wrapped around the magnetic shielding. We maintain a cell temperature of $70-80^{\circ} \mathrm{C}$, corresponding to an atomic density of $\sim 10^{11}-10^{12} \mathrm{~cm}^{-3}$.

A PBS and two photodiodes (PD) comprise the detection system. To create the EIT effect, the laser frequency is adjusted to the $D 1$ transition of ${ }^{87} \mathrm{Rb}(\lambda=794.987 \mathrm{~nm})$, i.e., $5 S_{1 / 2}, F=2 \rightarrow 5 P_{1 / 2}, F=1$, which is checked by observing
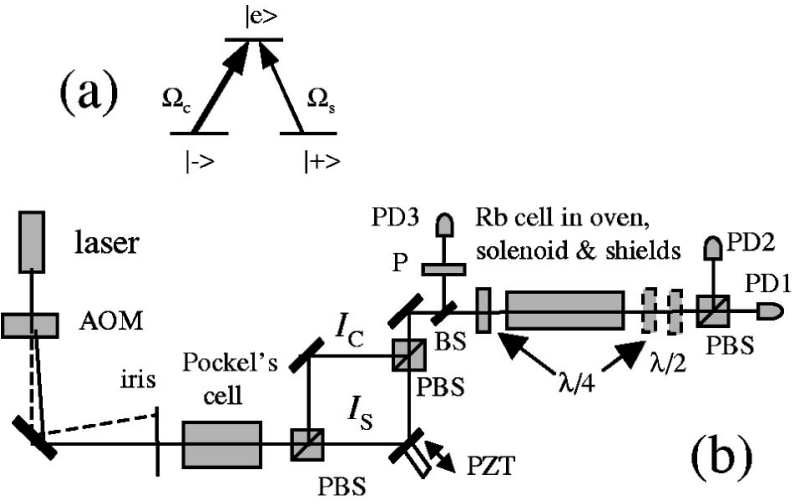

FIG. 1. (a) $\Lambda$ system of ${ }^{87} \mathrm{Rb}$ atomic states resonantly coupled to a control field $\left(\Omega_{c}\right)$ and signal field $\left(\Omega_{s}\right)$. (b) Schematic of the experimental setup. The intensity of the control field is $I_{c}$, the intensity of the signal field is $I_{s}$, PDs are photodiodes, BS is a beam splitter, and $P$ is a polarizer. Other components are defined in the text. 


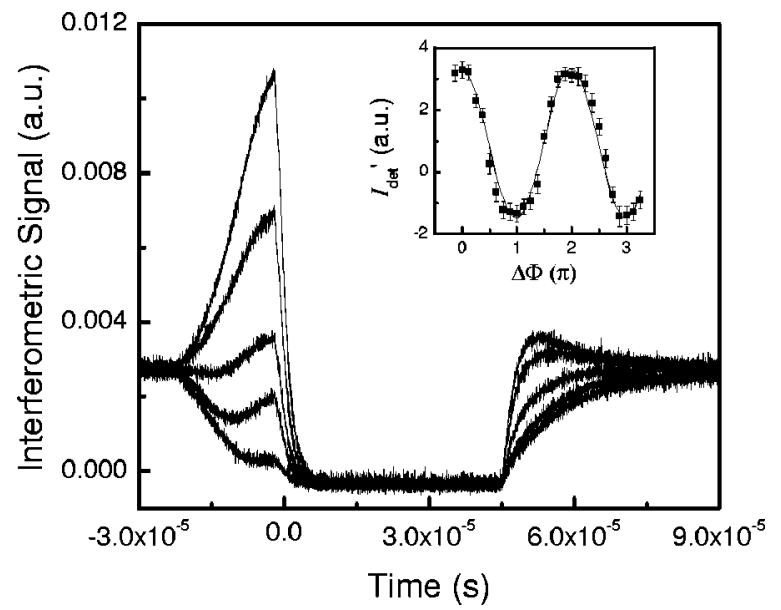

FIG. 2. Light storage interference patterns for different PZT voltages leading to different phase shifts between the control and signal beam. From top to bottom, for both prestorage and poststorage regions, the voltage changes from 90 to $150 \mathrm{~V}$ in $15 \mathrm{~V}$ steps. Inset: the detected signal intensity $I_{\text {det }}^{\prime}$ for varying PZT voltage after subtracting the control beam. The solid line is calculated from Eq. (1) with the detected part of the control beam, $I_{+}$, subtracted, $\Delta \Phi=\pi \Delta \mathrm{V} / 80 ; \Delta V$ is the voltage change of the PZT, with $80 \mathrm{~V}$ corresponding to a half-period phase shift of the signal beam.

the fluorescence spectrum. The laser beam diameter is about $\sim 5 \mathrm{~mm}$ with an output power of $\sim 8 \mathrm{~mW}(2.5 \mathrm{~mW}$ incident on the cell). For the light storage experiment, the time sequence of the control and signal fields is the same as in Ref. [7]. In the slowing experiment (where the signal pulse is delayed after propagation through the Rb cell), the control field is always present.

We would like to investigate the light storage for different relative phases between the control and signal beams. We do this for two purposes. First, the ability to maintain coherence in the atomic ensemble during storage is a prerequisite for storing an arbitrary polarization. Second, we would like to obtain a method that enables control of the coherence, but relies solely on modifying the input state. The alternative of using an external magnetic field [11] is media dependent. We hope that storage in different media [12] may benefit from this approach.

We introduce a relative phase by moving a PZT-controlled mirror in the Mach-Zender interferometer shown in Fig. 1(b). The phase shift corresponding to the mirror position has been calibrated by observing fringes. We use a $\lambda / 4$-plate before the $\mathrm{Rb}$ cell to cause the control beam to interfere with the signal beam. (For the linear case, we use a $\lambda / 2$ plate after the cell to generate our interference, instead of the $\lambda / 4$ plate before the cell.) Using this method, we can obtain both the phase and amplitude information of the signal beam from the interference pattern. The detected interference pattern at PD1 for a half-period phase variation is shown in Fig. 2. These patterns are a periodic function of the PZT voltage. The nonzero background intensity is from the control beam due to the $\lambda / 4$-plate rotation. This experiment clearly shows that the recovered light is from the signal beam, and that its phase has been maintained during the storage.

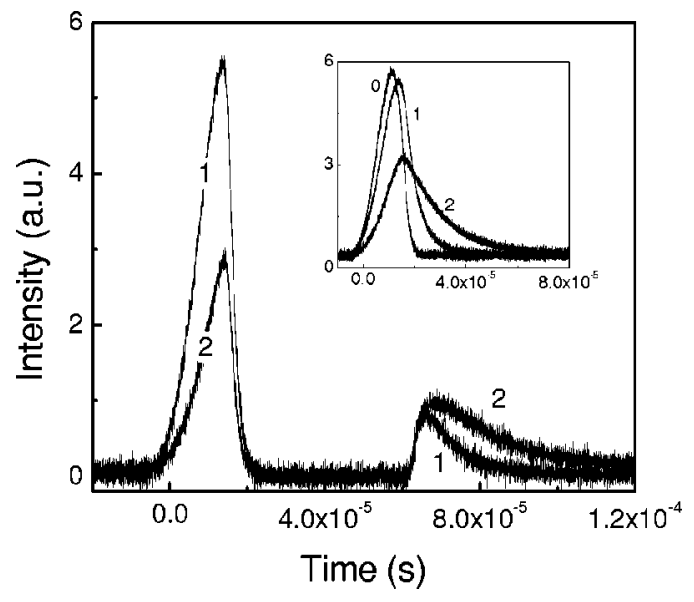

FIG. 3. Storage experiment for linearly (1) and circularly (2) polarized light. Inset: slowing experiment for linearly (1) and circularly (2) polarized light, line 0 is off the atomic resonance. In all cases, the leakage from the control beam has been subtracted.

To obtain the exact phase and amplitude information of the storage signal from these interference patterns, we write the expression for the photodiode signal as

$$
I_{\mathrm{det}}=I_{+}+I_{-}+2 \sqrt{I_{+} I_{-}} \cos (\Delta \Phi),
$$

where $I_{+}$is the intensity of the control beam that reaches PD1, $I_{-}$is the intensity of the stored signal that we wish to measure, and $\Delta \Phi$ is the phase difference between them. We control $\Delta \Phi$ using the PZT voltage, and can measure $I_{+}$directly by blocking the signal beam branch of the MachZender interferometer. (Note that the intensity $I_{-}$cannot be determined by blocking the control beam, because the signal beam is so weak that it is completely absorbed in the cell.) To verify that this method works correctly, we show an example for linear polarization in the inset of Fig. 2. The detected signal intensity (squares) is shown versus the phase shift after subtracting $I_{+}$. These data can be well fitted by Eq. (1), as shown by the solid line in the inset of Fig. 2. The results demonstrate that the storage signal intensity is not affected by the different phase shifts and confirm that the stored signal phase is maintained.

Now we turn our attention to the question of arbitrary polarized light storage. If we use a different polarization of light, a different atomic state will result. The depolarization and decoherence processes of the system determine whether or not arbitrary polarization can be stored. Depolarization is caused by the collisions of the target atoms with the cell walls or with other atoms and by external fields such as the leakage of the Earth's magnetic field. Decoherence is caused by the aforementioned effects as well as additional ones, including Doppler broadening and inhomogeneous magnetic fields [13]. Since the effects of these depolarization and decoherence processes vary for different atomic states, observation of $\sigma^{+}$storage [7] does not imply that storage of arbitrary polarization is possible. Conversely, the fidelity of storing different polarizations can be measured to obtain information about the decoherence processes. 
We begin by demonstrating the storage of linearly polarized light. Figure 3 (line labeled "1") is a typical storage result for linearly polarized light. For comparison, our $\sigma^{+}$ $-\sigma^{-}$result is also provided in Fig. 3 (labeled "2"). The latter is very similar to the result obtained in Ref. [7]. The maximum released signal intensity we obtained is about $40 \%$ of the input intensity after a 50- $\mu$ s time interval, depending on the readout control light intensity, atomic density, and external magnetic field. The amount of light recovered is generally governed by the amount of time the atoms spend in the laser beams; increasing the laser beam size gives a slightly longer storage time [14].

In our experiment (Fig. 3), we find that the prestorage signal intensity is about two times larger for linear light as compared to circular light. The observed ratio is proportional to the temperature of the sample. The smaller transmission line 2 can be explained by looking at the slowing experiment when the control beam is continuously turned on. As shown in the inset of Fig. 3, the pulse delay time of the circular polarization is larger than that of the linear polarization. A larger delay time leads to a narrower transparency window [6], which results in less transmission of circular light. The signal pulse duration in our experiment is about $15 \mu \mathrm{s}$, which corresponds to a $70-\mathrm{kHz}$-frequency width, while the transparency window for the $\sigma^{+}-\sigma^{-}$case under these conditions is measured to be about $40 \mathrm{kHz}$, in agreement with Ref. [7]. For the particular data shown, the signal after storage is larger for line 2 than for line 1 , however, this result depends on temperature.

We now expand our discussion to storage of elliptical polarization states. A $\lambda / 4$ plate is inserted before the $\mathrm{Rb}$ cell to generate the desired input polarization. Just as in the linearly polarized case, we modulate the PZT and confirm that the coherence of the signal beam is still maintained for other polarization cases. The interference intensities are measured by PD1, and the signal field components can then be determined just as the linear case. We then can determine the relative phase between the signal field and the control field by adjusting the PZT induced phase shift to zero, as determined from an interference pattern observed before the first $\lambda / 4$ plate (at PD3).

Note that we determine the amplitudes of the components of the control beam by blocking the signal field. We can also obtain the relative phase between these components by adding a second $\lambda / 4$ plate and a $\lambda / 2$ plate to the system, just before the PBS and detectors. Now each of the Stokes parameters of the control light (which give the coordinates on the Bloch sphere) can be directly determined from the detected light intensity, simply by rotating the $\lambda / 4$ and $\lambda / 2$ plates.

The relative phase of two signal field components can also be determined since we have measured the relative phase of two control field components. The normalized signal field components and their relative phase are shown in Fig. 4. We then compare these data to a calculation. The dotted line shows what the detected signal would look like when the polarization of the stored light was identical to the input light. The solid line adds the effect of paramagnetic Faraday rotation $[15,16]$ due to the population asymmetry in
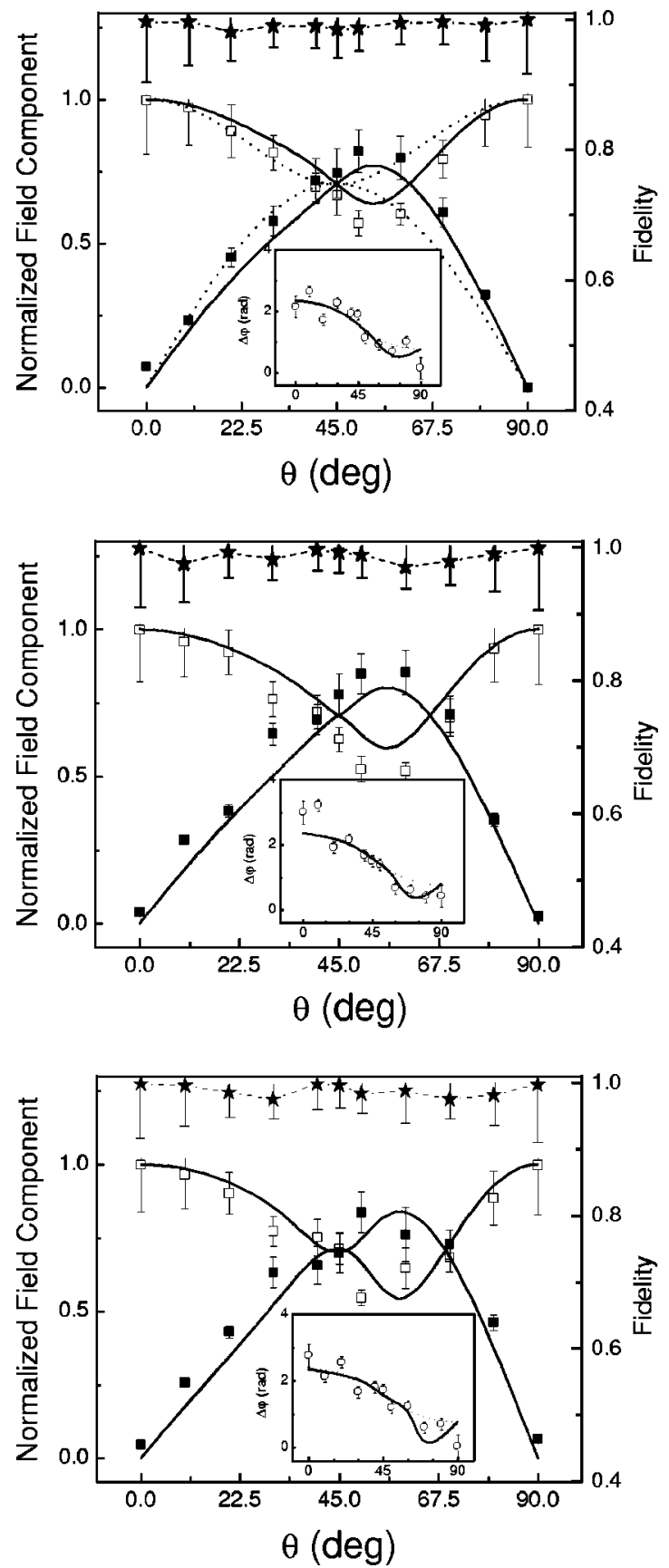

FIG. 4. The stored signal field components $E_{x}(\boldsymbol{\square})$ and $E_{y}(\square)$, and their relative phase $\Delta \varphi(\bigcirc)$ for input polarizations ranging from linear $(\theta=0)$, through elliptical and circular $\left(\theta=45^{\circ}\right)$ back to linear $\left(\theta=90^{\circ}\right)$. The angle $\theta$ is the rotation angle of the $\lambda / 4$ plate. Cell temperatures are (top) $69^{\circ} \mathrm{C}$, (middle) $74^{\circ} \mathrm{C}$, and (bottom) $80^{\circ} \mathrm{C}$. The fidelity $(\star)$ is calculated and the dashed line is a guide to the eye.

the magnetic substate of $\Lambda$ systems. This effect is estimated by calculating the populations from rate equations. The Faraday rotation causes the data to be asymmetric around the $\lambda / 445^{\circ}$ setting. We compare the solid line to the data points and calculate the fidelity [1]. Fidelity is defined as usual as $F=\left|\left\langle\psi_{\text {out }} \mid \psi_{\text {in }}\right\rangle\right|$ for a pure state, or more specifically for our system as $F=\left|\mathbf{E}_{\text {out }}^{*} \cdot \mathbf{E}_{\text {in }}\right|$. The results are shown in the same figure. All the polarization states are well maintained, al- 
though the intensities vary by up to a factor of 5 . The inset of Fig. 4 shows the phase difference between the electric-field components of the stored light pulse.

Due to the highly symmetric nature of our storage system, this set of measurements provides strong evidence that any arbitrary polarization could be stored. By rotating the initial $\lambda / 4$ plate from $0^{\circ}$ to $45^{\circ}$, we cover the points in a quarter circle of the Bloch sphere from linear to circular. The linear axis of the elliptical light, set by the input laser, has no physical effect on the cylindrically symmetric sample. Thus, we would have obtained the same result had that axis been different. This conclusion means that any point on one half of the Bloch sphere could be stored. Next, consider the effects of a parity transformation on the system. This also will have no effect on the measured results, but does reverse the helicity of the circular component of the elliptical light, giving us the other half of the Bloch sphere. Therefore our data for rotating the $\lambda / 4$ plate from $0^{\circ}$ to $45^{\circ}$ is enough to demonstrate that stored light of any polarization is possible.

In summary, we report the experimental storage of linear, elliptical, and circular polarized light in a vapor of $\mathrm{Rb}$ atoms. The fidelities show that the polarization state is well maintained during the storage. To make this measurement, we developed an all-optical method to determine the phase difference between the control and signal beam. This technique also allows us to control and determine the atomic coherence during storage, and should be applicable to other media. The ability to write an arbitrary polarization on an atomic ensemble is a necessary precursor for the realization of a quantum memory in an atomic vapor.

This work was supported by a Nebraska Research Initiative (NRI) grant. We thank S. X. Hu, D. F. Phillips, and S. F. Yelin for helpful and useful discussions, and B. Williams for his work on the apparatus.
[1] M.A. Hielsen and I.L. Chuang, Quantum Computation and Quantum Information (Cambridge University Press, Cambridge, 2000).

[2] See, e.g., S.E. Harris, Phys. Today 50(7), 36 (1997).

[3] M. Fleischhauer and M.D. Lukin, Phys. Rev. Lett. 84, 5094 (2000).

[4] J.R. Csesznegi and R. Grobe, Phys. Rev. Lett. 79, 3162 (1997).

[5] O. Kocharovskaya, Y. Rostovtsev, and M.O. Scully, Phys. Rev. Lett. 86, 628 (2001).

[6] M. Fleischhauer and M.D. Lukin, Phys. Rev. A 65, 022314 (2002).

[7] D.F. Phillips, A. Fleischhauer, A. Mair, R.L. Walsworth, and M.D. Lukin, Phys. Rev. Lett. 86, 783 (2001).

[8] C. Liu, Z. Dutton, C.H. Behroozi, and L.V. Hau, Nature (London) 409, 490 (2001).

[9] A.S. Zibrov, A.B. Matsko, O. Kocharovskaya, Y.V. Rostovtsev, G.R. Welch, and M.O. Scully, Phys. Rev. Lett. 88, 103601 (2002).
[10] D. Budker, D.F. Kimball, S.M. Rochester, and V.V. Yashchuk, Phys. Rev. Lett. 83, 1767 (1999).

[11] A. Mair, J. Hager, D.F. Phillips, R.L. Walsworth, and M.D. Lukin, Phys. Rev. A 65, 031802(R) (2002).

[12] A.V. Turukhin, V.S. Sudarshanam, M.S. Shahriar, J.A. Musser, B.S. Ham, and P.R. Hemmer, Phys. Rev. Lett. 88, 023602 (2002).

[13] D. Suter, The Physics of Laser-Atom Interactions (Cambridge University Press, Cambridge, 1997), Chap. 5.

[14] It is expected that the storage times can be significantly increased to about a second by the use of an antirelaxationcoated cell. See, e.g., D. Budker, V. Yashchuk, and M. Zolotorev, Phys. Rev. Lett. 81, 5788 (1998); J. Hager, D. Phillips, and R. Walsworth, Bull. Am. Phys. Soc. 47(3), 102 (2002).

[15] R.J. Knize, Z. Wu, and W. Happer, Adv. At. Mol. Phys. 24, 223 (1988).

[16] D.A. Van Baak, Am. J. Phys. 64, 724 (1996). 